\title{
El discurso del expresidente argentino Mauricio Macri con respecto a les migrantes: lo dicho y lo silenciado
}

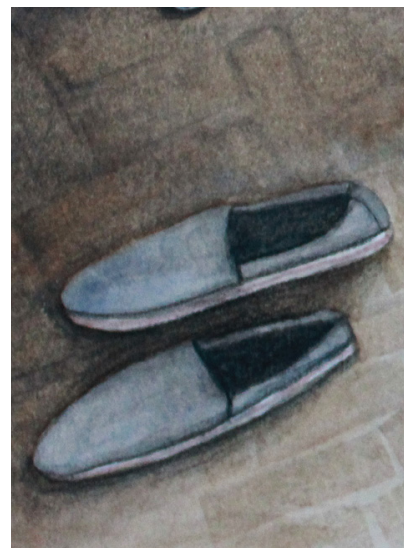

Rocío Flax

CONICET / Instituto de Lingüística, Facultad de Filosofía y Letras, Universidad de Buenos Aires, Argentina

rocioflax@educ.ar

Trabajo recibido el 28 de abril de 2020 y aprobado el 26 de julio de 2020.

\section{Resumen}

En este trabajo, analizamos la manera en que el expresidente argentino Mauricio Macri conceptualiza a les migrantes en sus alocuciones públicas durante el primer año de su mandato (diciembre 2015-diciembre 2016). Inscribimos el presente trabajo dentro del Análisis Crítico del Discurso (ACD) (Fairclough 1992, 2003, 2014; Hart 2014). Detectamos que ciertos temas o asociaciones - marcos conceptuales, en términos de la Lingüística Cognitiva(Lakoff 2002, 2010) — se reiteraban a lo largo de los discursos. De esta manera, pudimos agruparlos en cinco marcos: 1) Argentina como país de migrantes/inmigrantes europeos de principios de siglo XX; 2) relaciones comerciales, inversiones; 3) narcotráfico y terrorismo; 4) refugiados; 5) disimilación entre el in-group, los argentinos y el out-group, los extranjeros.

Palabras clave: migrantes, discurso político, marcos conceptuales, xenofobia, metáforas

\section{The discourse of former Argentine President Mauricio Macri regarding migrants: what was said and what was silenced}

\begin{abstract}
In this paper, we analyze the way in which Argentina's former president, Mauricio Macri, conceptualizes migrants in his public discourses during the first year of his term (December 2015-December 2016). We inscribe the present work within the framework of Critical Discourse Analysis (CDA) (Fairclough 1992, 2003, 2014; Hart 2014). We detected that certain themes and topics - conceptual frameworks, in terms of Cognitive Linguistics (Lakoff 2002, 2010) - were repeated throughout all the analyzed discourses.
\end{abstract}


We grouped them into five categories 1) Argentina as a country of European migrants / immigrants since the beginning of the 20th century; 2) business relationships, investments; 3 ) drug traffic and terrorism; 4) refugees; 5) dissimilation between the in-group, Argentines, and the out-group, foreigners.

Key Words: migrants, political discourse, conceptual frames, xenophobia, metaphors

\section{O discurso do ex-presidente argentino Mauricio Macri a respeito dos migrantes: o dito e o silenciado}

\section{Resumo}

Neste trabalho, analisamos o modo como o ex-presidente argentino Mauricio Macri conceitua os migrantes nos seus discursos públicos durante o primeiro ano do seu mandato (dezembro de 2015-dezembro de 2016). Inscrevemos o presente trabalho na perspectiva da Análise Crítica do Discurso (ACD) (Fairclough 1992, 2003, 2014; Hart 2014). Identificamos que certos temas ou associações -frames, em termos da Linguística Cognitiva (Lakoff 2002, 2010) - foram reiterados ao longo dos discursos. Dessa maneira, fomos capazes de agrupá-los em cinco marcos: 1) Argentina como um país de migrantes / imigrantes europeus desde o início do século XX; 2) relações comerciais, investimentos; 3) narcotráfico e terrorismo; 4) refugiados; 5) diferenciação entre o in-group, os argentinos, e o out-group, os estrangeiros.

Palavras-chave: migrantes, discurso político, frames, xenofobia, metáforas

\section{Introducción}

Durante la presidencia de Mauricio Macri (diciembre de 2015-diciembre de 2019), en Argentina, se sucedieron una serie de medidas de gobierno que perjudicaron a les ${ }^{1}$ migrantes, así como declaraciones de funcionarios que les valoraban de manera negativa. Esto, sumado a una política de seguridad interna que fomentó la violencia policial, generó un aumento de las actitudes violentas por parte de la policía hacia trabajadores migrantes.

En un trabajo anterior (Flax 2019a), analizamos la medida más importante del gobierno de Macri con respecto a las personas migrantes: el Decreto de Necesidad y Urgencia 70/2017, sancionado el 27 de enero del año 2017. Observamos cómo en dicho decreto se asocia a les inmigrantes tanto con el campo conceptual de los derechos como de la criminalidad. Por otra parte, para legitimar la urgencia de modificar la ley migratoria, ${ }^{2}$ se utiliza

1 El presente trabajo se encuentra escrito en lo que se ha denominado "lenguaje inclusivo". Para profundizar sobre las implicancias del mismo, ver Cardelli, 2018; Sarlo y Kalinowski, 2019.

2 La Ley de Migraciones 25.871 fue sancionada a finales del año 2003 durante la presidencia de Néstor Kirchner. Se trata de una ley que conceptualiza a la migración como un derecho humano y garantiza una serie de derechos sociales básicos para cualquier extranjero sin importar su situación migratoria. Además, incentiva la regularización de las situaciones migratorias ilegales por sobre la deportación. 
una serie de datos y estadísticas que, si bien son poco precisos, cumplen el objetivo de asociar a les migrantes con la inseguridad en general, el narcotráfico en particular, y la población carcelaria.

En este trabajo, analizamos la manera en la que el expresidente Macri conceptualiza a les migrantes en sus alocuciones públicas durante el primer año de su mandato, es decir, justo antes de la publicación del DNU mencionado. Inscribimos el presente trabajo dentro del Análisis Crítico del Discurso (ACD) (Fairclough 1992, 2003, 2014; Pérez, 2013, 2014; Flax, 2013; Hart, 2014; Martínez Romagosa y Flax, 2020).

En el próximo apartado, haremos una breve descripción del marco teórico para, luego, pasar a describir, en el apartado 3, la forma en la que hemos confeccionado el corpus y las herramientas metodológicas con las que fue analizado. En el apartado 4, desarrollamos el análisis.

\section{Marco teórico}

Para el ACD, el análisis debe comenzar por un problema social para observar el funcionamiento de los discursos vinculados con determinadas áreas de actividad y el papel que juegan en la producción y reproducción del poder. El discurso es visto como una práctica política e ideológica que, por lo tanto, entra en relaciones de interdependencia recíproca con otras prácticas sociales de manera que las representaciones que se incluyen en los discursos influyen en las actitudes, comportamientos y decisiones de las personas y las instituciones (Fairclough 1992, 2003, 2014).

$\mathrm{Al}$ interior del ACD, y partiendo de los mismos principios teóricos, encontramos diferentes corrientes de análisis que utilizan herramientas metodológicas diversas. Incluso, existen variantes que incluyen un mayor arraigo sociológico, histórico o cognitivo respectivamente. Sin embargo, la mayoría de las corrientes "mainstream" (Chilton, 2005) encuentran su filiación en la Lingüística Sistémico-Funcional (Hart, 2010; van Dijk, 1998) y toman de ella algunas de sus herramientas de análisis. Una de las consecuencias de esta filiación ha sido un análisis lingüísticamente muy riguroso y sistemático. Como contrapartida, durante varias décadas, algunas de sus herramientas no se desarrollaron o cuestionaron lo suficiente, a la vez que sus análisis tendieron a quedarse en un plano más bien descriptivo de la dimensión textual, obviando las explicaciones e interpretaciones que conectan el texto con lo social (Chilton, 2011; Hart, 2014).

Por ello, en los últimos años, algunos autores han buscado renovar las herramientas de análisis del ACD incluyendo elementos de la lingüística cognitiva. Autores como Christopher Hart $(2010,2014)$ y Paul Chilton $(2011,2014)$ proponen agregar a la dimensión descriptiva -mayormente desarrollada a través de la Lingüística Sistémico-Funcional- los aportes de la Lingüística Cognitiva para comprender los procesos de producción y comprensión de representaciones sociales, y los mecanismos de legitimación. En el próximo apartado, describiremos las herramientas 
metodológicas utilizadas en este artículo, que combinan los aportes basados en la Lingüística Sistémico-Funcional con la Lingüística Cognitiva.

\section{Conformación del corpus y metodología}

En primer lugar, se realizó una búsqueda léxica de los términos "migrante", "inmigrante", "extranjero", "extranjera", en todos los discursos del expresidente Mauricio Macri pronunciados entre diciembre de 2015 y diciembre de 2016, y subidos a la página web casarosada.gov.ar. Llamó nuestra atención la casi completa ausencia de dichos términos. "Inmigrantes" solo aparece para referirse a la inmigración europea de principios de siglo XX, mientras que "extranjeras" lo utiliza en referencia a empresas e inversiones, pero no con respecto a personas que deciden trasladarse a la Argentina.

Consideramos que Macri evita dichos términos como una forma de ser políticamente correcto. Así, por ejemplo, en lugar de "inmigrantes" o "extranjeros" utiliza términos como "habitantes del mundo". Entonces, buscamos en qué discursos hablaba de migrantes o personas provenientes de otros países (residan de forma temporaria o permanente en la Argentina o no) aunque no se los nombrara explícitamente.

El resultado de ambas búsquedas -aparición de términos "migrante", "inmigrante", "extranjero" y "extranjera", sumado a la referencia a migrantes o extranjeres (que no queda claro si residen o no en el país) a partir de ciertos rodeos, descripciones y alusiones ${ }^{3}$ - arrojó como resultado un corpus conformado por 23 discursos.

Una vez conformado el corpus, detectamos que ciertos temas o asociaciones - marcos conceptuales, en términos de la Lingüística Cognitiva (Lakoff 2002, 2010) - se reiteraban a lo largo de los discursos. De esta manera, pudimos agruparlos en cinco marcos.

(1) Argentina como país de migrantes/inmigrantes europeos de principios de siglo XX;

(2) Relaciones comerciales, inversiones;

(3) Narcotráfico y terrorismo;

(4) Refugiados;

(5) Disimilación entre el in-group, los argentinos, y el out-group, los extranjeros.

El corpus completo fue analizado a partir de cuatro categorías: marcos conceptuales (Lakoff 2002, 2010); estrategias referenciales (Hart 2010, 2014); estrategias predicacionales: tipos procesos y participantes asociados (Fowler et al 1983; Hodge y Kress 1993); y valoraciones (Martin y White 2005). En los fragmentos incluidos en el apartado 4, se verá reflejada la

3 Por ejemplo, al asociar "narcotráfico" con "control de fronteras" o con algo que debe ser echado del país. 
utilización de algunas de estas herramientas según las necesidades expositivas. A continuación, haremos una breve descripción de cada una.

En primer lugar, tomamos la Teoría de Framing (Lakoff 1987, 2002, 2010), según la cual cada vez que una persona utiliza el lenguaje para hablar de algo, activa marcos conceptuales en asociación con ese tema; en general, esto se realiza de manera automática e inconsciente, por lo tanto, no es algo que pueda evitar y, en muchas ocasiones, sucede sin que les hablantes se den cuenta y/o puedan controlarlo. Los marcos se organizan en sistemas de conceptos que se movilizan en conjunto, aunque sea parcialmente, en el momento en que se activa el marco. Por ejemplo, cuando pensamos o hablamos del concepto "vaca", se pueden activar conceptos como "rico", "asado", "alimentación", "leche", "inversión", "hierro", "proteína" todos asociados al marco que postula que la vaca es comida. O se pueden activar conceptos como "madre", "ternero", "amamantar", "pasto", "correr", si se activa el marco de que la vaca es una persona no humana. Cada vez que un marco conceptual se utiliza para hablar de cualquier tema - por ejemplo, la vaca como un objeto de producción y consumo-, la asociación entre ese tema y ese marco se fortalece.

Los marcos conceptuales, en algunos casos, se pueden estructurar a partir de metáforas (Lakoff 2002; Lakoff y Johnson 2003). Las metáforas conceptuales permiten comprender un concepto más abstracto o complejo por analogía con otro más simple o concreto. Por ejemplo, en occidente, el tiempo se piensa como dinero: se gana o se pierde tiempo, se invierte, se presta. De esta forma, la utilización de cada expresión metafórica da cuenta de un sistema conceptual subyacente, algunas veces tan convencionalizado que les hablantes no son conscientes de que están realizando una analogía.

En segundo lugar, utilizamos la propuesta de Hart (2010), quien analiza las estrategias referenciales (Reisigl y Wodak 2001) que se utilizan para denominar actores sociales. Para el caso de construcción de in-groups y out-groups en el discurso xenófobo da cuenta de las siguientes estrategias:

\begin{tabular}{ll}
\hline Estrategia & Medios lingüísticos \\
\hline Nacionalización & Gentilicios \\
\hline De-espacialización & Gentilicios \\
& Antropónimos \\
& Accionismos \\
& Topónimos metonímicos \\
\hline Disimilación & Metáforas de espacialidad \\
& Xenónimos \\
\hline Colectivización & Antropónimos \\
& Metáforas de espacialidad \\
\hline
\end{tabular}

Tabla 1: Estrategias referenciales según Hart (2010).

En tercer lugar, analizamos la distribución de procesos y participantes a partir del modelo de la Lingüística Crítica (Fowler et al 1983; Hodge y Kress 1993). El concepto de "proceso" —tomado de la Lingüística 
Sistémico-Funcional (Halliday y Matthiessen 2014) — refiere a aquello que posee una dimensión temporal. De esta forma, los procesos incluyen a los verbos, conjugados o en sus formas no finitas (gerundio, participio, infinitivo), pero también a las nominalizaciones. Por su parte, les participantes pueden ser relacionades de distintas formas con los procesos: pueden ser actores de los procesos, afectades, circunstancias o pueden estar conectades con otros objetos (entidades relacionantes). La cláusula típica, denominada "transactiva", está formada por dos participantes vinculades entre sí por un proceso. Estas cláusulas se caracterizan por el hecho de que une de sus participantes posee el rol de actore y le otre, de afectade. Las cláusulas no transactivas son aquellas que poseen une sole participante -actore o afectade- asociade a un proceso. Las cláusulas transactivas y las no transactivas componen lo que se denomina el "modelo accional" de representación. En cambio, el modelo relacional supone un tercer tipo de cláusula que establece una relación entre una entidad relacionante y otro elemento: las relacionales ecuativas sirven para establecer una identificación entre ambes participantes; las relacionales atributivas le adscriben características a la entidad relacionante; y las posesivas indican una relación de posesión.

Por último, utilizamos la Teoría de la Valoración (Martín y White 2005; Kaplan 2004), la cual describe y explica los sistemas de opciones semánticas que ofrece el lenguaje para evaluar, adoptar posiciones, negociar relaciones y construir personas textuales. El sistema de la valoración está compuesto por tres subsistemas: la actitud, el compromiso y la gradación. En este trabajo, nos concentramos en el subsistema de la actitud.

La actitud abarca tres regiones semánticas tradicionalmente vinculadas con la emoción, la ética y la estética (Martin y White 2005): el afecto da cuenta de las emociones positivas y negativas que expresan les actores sociales; el juicio se refiere a las valoraciones que se realizan sobre los comportamientos de las personas; y la apreciación supone la evaluación de fenómenos naturales y productos sociales. El siguiente cuadro grafica los diferentes tipos y subtipos de actitud considerados por Martin y White.

\begin{tabular}{|c|c|c|}
\hline \multirow{4}{*}{ Afecto } & \multicolumn{2}{|l|}{ Inclinación } \\
\hline & \multicolumn{2}{|l|}{ Felicidad } \\
\hline & \multicolumn{2}{|l|}{ Seguridad } \\
\hline & \multicolumn{2}{|l|}{ Satisfacción } \\
\hline \multirow{5}{*}{ Juicio } & \multirow{3}{*}{ Estima social } & Normalidad (¿cuán excepcional?) \\
\hline & & Capacidad (¿cuán capaz?) \\
\hline & & Tenacidad (¿cuán resuelte?) \\
\hline & \multirow{2}{*}{ Sanción social } & Propiedad (¿cuán étique?) \\
\hline & & Veracidad (¿cuán veraz?) \\
\hline \multirow{3}{*}{ Apreciación } & \multicolumn{2}{|c|}{ Reacción (¿cuánto atrajo nuestra atención?) } \\
\hline & \multicolumn{2}{|c|}{ Composición (referida a proporciones y detalles) } \\
\hline & \multicolumn{2}{|c|}{ Evaluación (¿qué importancia social otorgamos a lo evaluado?) } \\
\hline
\end{tabular}

Tabla 2: Subsistema de la actitud. 


\section{Análisis}

\subsection{Argentina, un país de inmigrantes de Europa}

Macri utiliza el término "inmigrante" solo en referencia a las migraciones europeas de fines del siglo XIX y, especialmente, de principios del siglo XX. En este sentido, les migrantes son algo del pasado, ya no existen. No se dice, por ejemplo, que haya italianos en Argentina, sino hijos de italianos. En referencia a les migrantes actuales, emplea otros términos, por ejemplo, "comunidad japonesa".

Por otra parte, el expresidente refuerza una representación que forma parte del sentido común argentino, que considera que todes les "ciudadanes" argentines son hijes de inmigrantes, en particular de inmigrantes europees. En esta serie de discursos, Macri plantea una división entre nosotres, les argentines y elles, les inmigrantes, pero siempre para marcar la relación de filiación entre ambos grupos.

Les inmigrantes europees son asociades al trabajo duro y al emprendedurismo (neologismo que suena muy extemporáneo para referirse a migrantes de hace más de 100 años), es decir, se activa la representación —con bastante circulación en el país- de la cultura del trabajo, de le inmigrante que llegó al país sin nada, pero logró una vida mejor a partir de su propio esfuerzo:

(1) Pasaron 18 años de la última visita de un Primer Ministro a la Argentina, es realmente increíble que haya pasado tanto tiempo, porque no sé si hay un país con el cual tengamos semejante nivel de vínculos afectivos, culturales. En la Argentina, Matteo, cuesta encontrar un ciudadano que no tenga algún abuelo, bisabuelo, padre, italiano; en mi caso todos. Y se refleja en nuestra cultura, en nuestros artistas, en nuestra arquitectura, en nuestra gastronomía, en nuestra forma de apasionarnos ${ }^{4}$ con las cosas que hacemos todos los días. (...) Creemos que así como mi abuelo llegó a este país y este país le abrió las puertas para crecer y desarrollarse, él era de Polístena, en el sur de Italia - hace poco fui a visitar donde él nació, fue un momento conmovedor para mí-queremos que la Argentina vuelva a abrir sus puertas para otros italianos vengan a ayudarnos a crecer y que juntos podamos generar esas oportunidades (Discurso por la visita a la República Argentina del Primer Ministro de Italia, 16/2/2016) (cursivas nuestras).

En el fragmento (1), la inmigración es una herencia cultural omnipresente ("se refleja en nuestra cultura, en nuestros artistas, en nuestra arquitectura, en nuestra gastronomía, en nuestra forma de apasionarnos con las cosas que hacemos todos los días"), pero, como dijimos, una cuestión del pasado. No hay italianes, hay hijes, nietes, bisnietes de italianes.

Macri se pone como ejemplo de argentine descendiente de italianes e incluye el recurso del story-telling para provocar el interés y la adhesión emocional de sus interlocutores: relata la historia de vida de su abuelo y 
su propia visita al lugar de nacimiento incorporando valoraciones afectivas ("vínculos afectivos", "fue un momento conmovedor para mí").

El país se representa a través de la metáfora del edificio: es una casa con sus puertas abiertas. La expresión "vuelva a abrir sus puertas" implica, por un lado, que ya lo hizo en el pasado (a principios del siglo XX) y luego las cerró. Pero, por otro lado, plantea una diferencia con la situación anterior. Ahora, Argentina no abre las puertas a les italianes para que vengan a vivir, sino para que vengan a invertir dinero. De hecho, la relación parecería invertirse: antes les italianes venían porque necesitaban ayuda ("crecer y desarrollarse") y les argentines se la proporcionaron. Ahora, les argentines necesitan ayuda (económica o financiera) y se la piden a les italianes: "abrir puertas para otros italianos vengan a ayudarnos a crecer".

En el fragmento (1), vemos, por último, que prevalecen las estrategias de diferenciación entre nosotres, les argentines y elles, les italianes (aun a pesar de las referencias afectivas y familiares), que solo se cierra al final del pasaje y en referencia a la economía argentina: "que juntos podamos generar esas oportunidades".

(2) Y yo digo que en este país hay un gigantesco espíritu emprendedor, y tengo mi teoría personal del por qué: yo me puse a pensar en la campaña, durante esos dos años en que recorrimos todo el país, y me decía: ¿Por qué tantas iniciativas en todos lados, tanta gente con ganas de emprender ideas? Es obvio para mí: es porque nosotros venimos de nuestros abuelos, de nuestros padres, que en una época donde no teníamos las herramientas de hoy -ellos no podían chatear con un amigo en América del Sur, ni por Facebook ver una foto-, igual decidieron subirse a un barco más de 30 días para emprender viaje hacia un lugar que se llamaba América, a "hacer la América” (Acto de anuncio del paquete de proyectos de ley para emprendedores y empresas, 18/8/2016) (cursivas nuestras).

En el fragmento (2), los atributos de "la cultura del trabajo" también se encuentran actualizados, aggiornados al discurso neoliberal: "emprendedor", "iniciativa", "emprender" se repiten en varias ocasiones. El espíritu emprendedor de les argentines tiene, entonces, su causa en la herencia cultural europea: una vez más, se activa la representación de que Argentina es un país de inmigrantes europees al caracterizar al nosotres, les argentines, en general, como hijes o nietes de europees: "Es obvio para mí: es porque venimos de nuestros abuelos, de nuestros padres". Dentro de esta actualización de vocabulario para referirse a les migrantes, observamos una banalización o incomprensión de la historia al referirse a la inexistencia de Facebook para determinar el valor de inmigrantes que, como dijimos, eran forzados a migrar por las guerras y la pobreza de sus países de origen.

Por su parte, "Hacer la América" es una frase muy utilizada desde principios del siglo XX para referirse a les europees que venían a América a hacer fortuna (Moliner 2007) y luego tenían planificado regresar a su país (Discepolo [1925] 2006). Este sentido tiene un matiz negativo, de aprovechamiento, desagradecimiento, etc. No obstante, es una metáfora que puede tener el significado de construcción de un continente (sobre todo considerando el imaginario de la Argentina como un territorio desierto, necesitado de la 
civilización europea para desarrollarse) que se utiliza en referencia a la voluntad de trabajo con que vinieron les europees.

Veamos un último ejemplo donde el expresidente asocia la migración a un suceso del siglo pasado:

(3) Y en eso estamos, en trabajar todos los días para remover obstáculos, destrabar, simplificar todo lo que haga al crecimiento, a la producción, al desarrollo, porque -insisto- el camino para construir la Argentina que soñamos, esa Argentina en la cual yo estoy comprometido con mis compatriotas, es la del trabajo. Es recuperar la cultura del trabajo que trajeron nuestros antepasados españoles que vinieron a radicarse en la Argentina, así como italianos, polacos, ucranianos o lo que usted quiera encontrar; porque, la verdad es que, si hay algo de bueno que tiene nuestro país, es la enorme diversidad cultural que hemos tenido siempre (Apertura del Encuentro empresarial iberoamericano, realizado en Argentina, 25/4/2016) (cursivas nuestras).

En el fragmento (3), se repiten las mismas representaciones. En primer lugar, Macri vuelve a clasificar a les argentines como descendientes de europees ("nuestros antepasados españoles (...) así como italianos, polacos, ucranianos o lo que usted quiera"). La insistencia en esta representación lo habilitará, como veremos en el último apartado, a separar a les argentines de los pueblos originarios.

En segundo lugar, asocia, una vez más, a les inmigrantes europees con "la cultura del trabajo". Esta representación es, en Argentina, casi un estereotipo. La cultura del trabajo, referida a les inmigrantes de principios de siglo $\mathrm{XX}$, les muestra huyendo de la guerra y la miseria en Europa, llegando en barcos casi sin nada, trabajando en general en empleos duros, construyendo sus propias casas, saliendo de la miseria a partir de su propio esfuerzo y sacrificio (Flax 2019b). En el discurso de Macri, este estereotipo se menciona de manera explícita ("recuperar la cultura del trabajo que trajeron nuestros antepasados españoles") y también se evoca a partir de juicios de capacidad y tenacidad referidos a les migrantes: "remover obstáculos", "destrabar", "simplificar", "crecimiento", "desarrollo", "producción", "trabajo".

En un discurso del 21 de noviembre de 2016 - en el marco de una declaración conjunta del presidente Mauricio Macri y del Primer Ministro de Japón, quien se encontraba de visita en la Argentina- la comunidad japonesa en Argentina también es caracterizada por el trabajo esforzado y la superación: "de la cual siempre también aprendimos la importancia de la cultura del trabajo; la humildad en la búsqueda de la superación permanente, que en el tiempo dio lugar al Kaizen, la mejora continua". Es decir que "la cultura del trabajo" no sería algo natural de les argentines, sino importado (de hecho, al parecer la perdieron como se implica a partir del verbo "recuperar" en el fragmento (3)). Esto también puede asociarse con otro estereotipo, en este caso de les argentines como vagues, chantas, etc.

Por último, en el fragmento (3), aparece la representación de la Argentina como un crisol de razas, metáfora ampliamente utilizada dentro de la 
historia argentina y actualizada con las posturas multiculturalistas (Golay 2013; Soria 2009), que aparece en el discurso de Macri a través de la expresión "una enorme diversidad cultural". Por supuesto, la diversidad cultural suele ser vista, como en este discurso, como algo positivo per se, sin cuestionarse, por ejemplo, sobre la convivencia de esas culturas ni las desigualdades sociales y económicas que las atraviesan. ${ }^{5}$

\title{
4.2. Criminalidad: el narcotráfico
}

Un conjunto de discursos del expresidente asocia, de manera implícita, "el narcotráfico" y "el terrorismo" (estrategias referenciales que abstraen a les actores sociales) con les extranjeres. Por ejemplo, en el fragmento (4), Macri genera el sobreentendido de que el narcotráfico viene de afuera (y de países de América) porque lo considera un caso de "seguridad nacional" y combatirlo (marco conceptual de la guerra) le corresponde a gendarmería, que es la fuerza de seguridad que se ocupa de las fronteras terrestres. Aquí los pronombres se utilizan como estrategias referenciales de disimilación ("nuestro país", "nuestra seguridad") frente a une otre amenazante. Como plantean Hart (2010) y Lakoff (1987), la estrategia de asociar extranjeres con narcotráfico habilita un desplazamiento metonímico, donde del hecho de que algunes migrantes sean criminales o narcotraficantes, se termina asociando a que todes les migrantes son criminales o narcotraficantes:

\begin{abstract}
(4) Los gendarmes son muy importantes para nuestro país, muy importantes para nuestra seguridad nacional, para el combate del narcotráfico y entonces quiero mandarle [sic] las condolencias a sus familias, a todo el cuerpo y les pido a todos que hagamos un minuto de silencio por esas familias. Gracias. (Cierre de la Conferencia anual de la Unión Industrial Argentina, 14/12/2015) (cursivas nuestras).
\end{abstract}

(5) También están los intercambios en educación y en otros aspectos de la seguridad, porque ustedes tienen una gran experiencia en todo lo que es seguridad de fronteras y en esta lucha contra el narcotráfico y el crimen organizado es muy importante también trabajar en conjunto con ustedes y que nos ayuden a mejorar lo más rápido posible, en ese aspecto también. (Conferencia de prensa por la visita a la República Argentina del primer ministro de Canadá, 18/11/2016) (cursivas nuestras).

En el fragmento (5), Macri vuelve a vincular el narcotráfico y el crimen organizado con las fronteras. Es decir, que el narcotráfico y el crimen organizado son algo que viene de afuera, algo que entra, una amenaza o un peligro para la Argentina desde el exterior. Como podemos observar, la activación de marcos conceptuales bélicos es recurrente en el discurso del expresidente:

5 Las posturas multiculturales acríticas valoran la contribución de los distintos grupos étnicos y migrantes al crecimiento y desarrollo del país, promueven el respeto y la tolerancia a la diversidad cultural para la disminución de la discriminación y los prejuicios y reconocen derechos de igualdad formal, al tiempo que mantienen inalterada la estructura de poder que (re)produce las condiciones materiales y simbólicas de dominación y exclusión social (Soria 2009): "Es preciso asumir que las diferencias culturales esconden relaciones de poder, y que esta idea de una coexistencia armónica y horizontal, no conflictiva y no jerárquica, de formas o grupos culturales, es una de las trampas del multiculturalismo, que esconde muy concretas y violentas situaciones de explotación, exclusión y discriminación.” (Stang 2009, 342). 
(6) Y siempre acuérdense, que los argentinos unidos, trabajando codo a codo, como mostramos hoy, sin importar el partido político al cual pertenezcamos, sino teniendo en cuenta qué es lo que necesita el argentino, que es trabajo, oportunidades, seguridad, combatir - que es el tercer compromiso- el narcotráfico y echarlo de este país, eso lo vamos a lograr juntos, juntos, juntos somos imparables. (Discurso en el marco de la visita a la provincia de Jujuy, 5/2/2016) (cursivas nuestras).

En el fragmento (6), la asociación entre narcotráfico y extranjeros se debe inferir a partir de la frase "echarlo del país". De manera explícita, solo se menciona al in-group "los argentinos", que se oponen al narcotráfico. La acción de sacar al narcotráfico de Argentina activa la metáfora del país como un tipo de recipiente, un edificio o una casa, en el cual se encuentra une intruse que traspasó los límites, que no pertenece a ese espacio y, por lo tanto, debe ser expulsade.

\subsection{Visión utilitarista: trabajo, empresas, inversiones y turismo}

En otros discursos, el expresidente se refiere a les extranjeres en términos utilitarios. Lo que pide Macri — como observamos en el fragmento (1) — es que vengan empresas a invertir o personas a trabajar para que el país se desarrolle. En algunos casos, no hay mayores especificaciones, pero cuando se mencionan nacionalidades, estas personas o empresas provienen de Norteamérica, Europa o ciertas partes de Asia. No se valora el trabajo de les migrantes latinoamericanes, por ejemplo. Dentro de estos discursos, también aparece el turismo - en términos de personas que vienen a conocer o visitar la Argentina- como un beneficio para el país.

(7) En este país, ya hay una enorme participación de la comunidad española: es el segundo país inversor en la Argentina. Son más de 250 empresas que están acá, que han sobrevivido a las distintas crisis que hemos tenido que sufrir. (...) Y esta es la tarea fundamental de mi Gobierno: generar trabajo. Por eso hoy estoy acá, para decirles que son muy bienvenidos, que queremos que sean parte de este proceso; que sabemos que ya tienen una participación importante en la banca, en las telecomunicaciones, en la energía, en la construcción, en los seguros. Queremos que la sigan teniendo y que la amplíen, porque más allá de que está todo cruzado por la sociedad del conocimiento, de la innovación, y ahí es donde apostamos mucho con los talentos, con los recursos humanos valiosos que tiene la Argentina, gracias a los cuales no podemos prever - por suerte-lo que seremos capaces de crear. Pero ya hay, de movida, algunos sectores estratégicos donde necesitamos el apoyo de sus empresas. Necesitamos que ustedes se involucren, inviertan, apuesten y participen. Me refiero a la energía, a la minería sustentable, al turismo, a la industria de la alimentación, y a la infraestructura (Apertura del Encuentro empresarial iberoamericano, realizado en Argentina, 25/04/2016) (cursivas nuestras).

En general, en Argentina, "comunidad española" o "colectividad española" -o de cualquier otro país - hace referencia a las personas que nacieron en dicho país y vinieron a vivir a la Argentina, y a sus descendientes. Además, les migrantes españoles constituyen un grupo muy numeroso desde fines de siglo XIX. Por lo tanto, a pesar del contexto situacional (Encuentro Empresarial Iberoamericano) y de estar hablando de empleo e inversiones, la primera oración del fragmento genera una expectativa que se contrapone 
a lo que sigue: "comunidad" no refiere a personas que viven en la Argentina, sino a inversores o, directamente, a la nominalización inversiones. De hecho, les actores sociales de este fragmento se encuentran mayormente abstraídes al ser representades como empresas, talentos o recursos humanos.

Les españoles son bienvenides al país, pero no a vivir —es decir, no invita a migrantes- sino como empresaries. Elles forman parte de la Argentina no porque viven, trabajan y contribuyen, de esta forma, con el país sino porque son dueñes de acciones (proceso posesivo): "ya tienen una participación importante en la banca, en las telecomunicaciones, en la energía, en la construcción, en los seguros".

La Argentina se representa como un país no desarrollado, que posee fuerza de trabajo (las personas son cosificadas al representarlas como "recursos humanos"), pero no posee dinero o tecnología y, por lo tanto, necesita (proceso repetido en dos ocasiones) ayuda de les empresaries españoles. Así, la diferenciación entre un nosotres y un ustedes, que Macri construye en todo el fragmento a través de pronombres y desinencias verbales, corresponde al gobierno ${ }^{6}$ y les empresaries extranjeres.

(8) Y estaremos felices de recibir otros alemanes, que quieran venir a vivir a nuestro país y nos ayuden a construir la Argentina que soñamos (Conferencia en la Fundación Konrad Adenauer, Alemania, 5/7/2016).

La metáfora de la casa se activa, en el fragmento (8), a partir de la valoración de afecto "felices de recibir". La distinción entre dos grupos es marcada a lo largo de toda la breve cita ("estaremos", "nuestro", "nos", "soñamos" vs. "alemanes", "quieran venir", "ayuden"). En este caso, sí parecería que habla de migrantes y no de empresas o inversiones. No obstante, la visión utilitarista se mantiene porque las personas que son bienvenidas al país son aquellas que colaboren con su crecimiento. En este sentido, se supone que la inmigración (¡alemana ${ }^{7}$ !) es positiva y no se la conceptualiza como una carga económica (otra representación del sentido común sobre la inmigración, Wodak 2003), con la condición de que quienes vengan lo hagan para ayudar a los nativos.

(9) Por eso, hoy, de la mano del testimonio, que ustedes dan, como Presidente viajo por el mundo invitando a todos los habitantes de este planeta a que vengan, a que nos conozcan, a que participen, a que trabajen en conjunto con nosotros, que se asocien y que nos ayuden a hacer crecer a nuestro país (Acto de despedida a los "Cascos Azules”, quienes brindarían asistencia en Haití, 10/10/2016).

Como dijimos al inicio del trabajo, Macri evita utilizar términos como “(in)migrante" o "extranjere", términos que remitirían a estrategias de

6 "Las distintas crisis que hemos tenido que sufrir" podría referir a todes les argentines. No obstante, si bien podría ser de su interés, "queremos que sean parte de este proceso; que sabemos que ya tienen una participación importante en la banca, en las telecomunicaciones, en la energía, en la construcción, en los seguros. Queremos que la sigan teniendo y que la amplíen" referiría al gobierno.

7 Macri invita a alemanes en consonancia con el ideal de la inmigración buscada en Argentina desde el siglo XIX. No hay un discurso equivalente en el cual invite a personas de países pobres, personas que podrían necesitar migrar por razones políticas, económicas, etc. 
disimilación y de-espacialización. En este fragmento, utiliza el eufemismo políticamente correcto "todos los habitantes de este planeta". No obstante, la división nosotres/elles se mantiene en términos como "vengan", "nos conozcan", "participen", "trabajen con nosotros", "nos ayuden", "nuestro país".

Una vez más, el país es representado como una casa con sus puertas abiertas, pero no está claro que el expresidente invite a otras personas a vivir en el país o solamente a invertir. Así, "participen", "trabajen" e, incluso, "ayuden a hacer crecer nuestro país" podría referir tanto a migrantes como a empresas, pero "se asocien" remite definitivamente a relaciones entre empresaries.

En todos los fragmentos citados en este apartado, Macri representa a la Argentina como un país que necesita ayuda; la ayuda de afuera es bienvenida, no es un problema ni una amenaza. No obstante, prevalece la referencia a empresas o personas de países desarrollados y no queda en claro que se esté fomentando la radicación en el país y no solamente la inversión económica. De esta manera, la migración no es conceptualizada como un derecho de las personas (como indica la Ley de Migraciones de la Argentina que rige desde el año 2003), sino como una utilidad para el país.

\subsection{Crisis de refugiades}

En varios discursos, Macri hace referencia a refugiades o a la "crisis de los refugiados", pero no profundiza en la problemática. Ya denominarlos "refugiades", en lugar de "(in)migrantes", es aceptar los términos europeos para describir a las personas que se ven obligadas a dejar su país de nacimiento. La distinción "refugiade"/"migrante" no es un dato de la realidad. Gran parte de las migraciones son forzadas ya que son la consecuencia de condiciones donde la vida está en peligro: ${ }^{8}$ pobreza extrema, persecuciones políticas o, como en Siria, guerras. De hecho, fueron guerras las que trajeron a les "inmigrantes" (no conceptualizades como "refugiades") europees durante principios y mediados del siglo XX a la Argentina.

“(In)migrante" y "refugiade" implican dos marcos conceptuales diferentes para referirse a personas que dejan su país para vivir en otra parte del mundo. El término "migrante" corresponde a lo que Hart (2010) denomina un "accionismo", en tanto clasifica a le actore social por una acción que este realiza, cambiar de lugar; mientras que "refugiade" plantea a la acción de conseguir refugio como ya lograda, ya acogides por algún país (aunque no siempre es el caso), pero además les representa no desde un punto de vista agentivo, sino en el rol de afectades (alguien les refugia y lo hace porque se encontraban en peligro) o como un atributo más o menos estable que les caracteriza. "Refugiades", frente a "(in)migrantes" reduce el poder y control sobre sus vidas que poseen las personas que dejan su país de origen.

8 De hecho, se podría decir que todes les migrantes responden a situaciones extremas, ya que este término no es utilizado para referirse a, por ejemplo, profesionales que eligen desarrollar su carrera en otros países (Domenech 2009). Según Domenech, "extranjere" refiere a un estatus jurídico, pero "migrante" a una condición social. 
(10) los argentinos sentimos que tenemos capacidad y vocación de contribuir en las soluciones a los distintos desafíos que existen en estos tiempos que vivimos. $Y$ en estos desafíos, que también comparte en su agenda usted como Secretario General, el tema de la crisis humanitaria de los refugiados, el cambio climático, el mantenimiento de la paz, son algunas de las tareas, junto, por supuesto, a siempre trabajar por la mejora de las instituciones, su transparencia, luchar contra la violencia de género, como hablábamos hace un instante. Es una agenda vasta de cosas en la cual la Argentina ya ha empezado a contribuir, como ha mostrado en su vocación de recibir refugiados de Siria, con todo lo que están viviendo, nosotros ya tenemos una comunidad siria importante en la Argentina. Como también ayudar en el proceso de paz a los hermanos colombianos; tenemos capacidad técnica para trabajar en la inclusión social, en la capacitación, en resolver el tema de las minas. Son varias las cosas en las que podemos contribuir y ya nos hemos comprometidos a hacerlo (Declaración por la visita a la República Argentina del Secretario General de las Naciones Unidas,8/8/2016) (cursivas nuestras).

El expresidente enumera una serie de problemas ("desafíos") globales en los que Argentina colabora o quiere colaborar: "los argentinos sentimos que tenemos capacidad y vocación de contribuir en las soluciones a los distintos desafíos que existen en estos tiempos que vivimos", "el tema de la crisis humanitaria de los refugiados, el cambio climático, el mantenimiento de la paz, son algunas de las tareas". Dentro de esta enumeración rápida de diferentes "temas", inserta el problema de les refugiades, como un caso en el cual ya ha intervenido. La referencia a la situación en Siria que generó la mencionada "crisis humanitaria" se da a través de una frase por completo inespecífica ("con todo lo que están viviendo"), que representa la guerra civil en Siria a partir de un verbo existencial, donde no hay actores responsables, sino tan solo una experiencia que sufren les siries (algo similar sucede con la expresión "crisis humanitaria" donde no hay lugar para procesos o participantes que sean responsables). Además, "todo" es un pronombre indeterminado que no da ninguna pista sobre qué están viviendo las personas en Siria, ni cómo lo considera o evalúa Macri.

(11) Hemos hablado del compromiso en conjunto contra el cambio climático, el compromiso que hemos asumido en la última Cumbre en París, y que estamos ratificando por el Congreso de la Nación; el compromiso solidario a trabajar con el tema de los refugiados, que son más de 60 millones, nos contaba recién Ban Ki-moon. El compromiso a trabajar contra la violencia de género, y hay una buena noticia: las Naciones Unidas abrirá una Oficina de la Mujer en la Argentina, y eso nos va a permitir desarrollar planes conjuntos a través de nuestra Canciller (Declaración por la visita a la República Argentina del Secretario General de las Naciones Unidas,8/8/2016) (cursivas nuestras).

Macri despersonaliza a les refugiades: en el fragmento (11), son un tema y un número. Además, borra cualquier profundidad política con respecto a la problemática de las personas que se ven forzadas a abandonar su país al valorar la acción de Argentina (nominalizada: "compromiso") como solidaria. La ayuda a les refugiades se conceptualiza como un asunto de "solidaridad" (definido por la RAE como la "adhesión circunstancial a la causa o a la empresa de otros") y no, por ejemplo, como un asunto de justicia o responsabilidad en tanto país miembro de las Naciones Unidas. Es decir, el marco de la solidaridad excluye otras posibles formas de considerar el 
tema: la política, la guerra, las relaciones internacionales. Asimismo, supone que es un problema ajeno a la Argentina, que, en principio, no la involucra o no le compete: se trata de un problema de otro, en el cual la Argentina interviene voluntariamente.

\subsection{Construcción de in-group vs. out-group: la disimilación}

(12) Eso es lo que todos queremos, que el aporte de cada uno de nosotros cuente, pese, sea reconocido, sea importante para que otro argentino $u$ otro pueblo originario puedan crecer y desarrollarse (Encuentro con pueblos originarios en Buenos Aires, 17/12/2015).

En el fragmento (12), Macri separa a "argentino" de "pueblo originario" (además compara personas con una abstracción: pueblo) señalando que se trata de dos categorías diferentes de personas. Esto constituye una estrategia de disimilación que, como dijimos en el primer apartado de análisis, se encuentra habilitada por la insistencia en la representación de que les argentines descienden de europees y, entonces, no descienden de indígenas americanes o "pueblos originarios".

(13) La verdad que estoy muy contento porque estamos todos acá reunidos, muchas caras conocidas, muchos que hemos hablado sobre este tema, durante mucho tiempo, en distintas ocasiones y creo que es central porque el tema es central, fundamental. Yo diría que es casi único en las vidas de todas las personas, sean argentinos o habitantes de otras partes del mundo, pero acá estamos para resolver el tema de los argentinos y es que estemos acá comprometidos finalmente y definitivamente para trabajar en serio para mejorar nuestro sistema de salud (Acto de presentación de reformas para una mejora integral del sistema de salud, 2/o8/2016) (cursivas nuestras).

En el fragmento (13), Macri realiza una división innecesaria para hablar del tema de salud: separa a les argentines (in-group) de les "habitantes de otras partes del mundo" (out-group). El expresidente explicita que no le interesa resolver los problemas de les habitantes de otras partes del mundo ("pero"), se encuentra para resolver el problema de les argentines. Todo esto puede parecer una obviedad, de hecho, lo es: el presidente de Argentina se ocupa de los problemas de les argentines, no de los problemas de otros países, y a toda la humanidad le interesa su salud. Justamente por ser una obviedad resulta significativo que lo aclare: podría haber dicho "un tema fundamental para todes", "un tema fundamental para todo el mundo", pero elige realizar la separación entre dos grupos, que de esta manera se alejan (estrategia de disimilación).

Si pensamos que "habitantes de otras partes del mundo" ${ }^{9}$ no refiere necesariamente a personas que, efectivamente, en el presente de la enunciación habitan países que no son Argentina, sino que se trata de un eufemismo para no decir "inmigrantes" o "extranjeres", implicar que no es responsabilidad de Macri resolver los problemas de salud de les no argentines puede llevar a activar otra representación del sentido común en Argentina que 
plantea que les extranjeres llegan al país para abusar del sistema gratuito de salud. En otros discursos, la misma estrategia de disimilación, en la que queda ambiguo si se refiere a personas que viven en otros países o que vienen a vivir a la Argentina, se utiliza para referirse a la competencia por tener un trabajo:

\begin{abstract}
(14) Tenemos que poder ser capaces de sentarnos permanentemente alrededor de una mesa para discutir cómo mejoramos, porque el mundo nos abre una mano para invitarnos, quiere venir a invertir, pero también el mundo es enorme y hay productores de todo lo que hacemos que compiten con nosotros, que compiten con nuestra capacidad de generar trabajo, porque ellos también necesitan trabajo (20/5/2016) (Discurso por el $162^{\circ}$ aniversario de la Bolsa de Cereales de Buenos Aires, cursivas nuestras).
\end{abstract}

Así, en el fragmento (14), no se referiría a personas que viven en la Argentina, sino más bien a la competencia entre países productores de determinados bienes. No obstante, evoca la representación del sentido común de que hay una competencia con les extranjeres por un trabajo que no alcanzaría para todes. Otras personas, otres productores, entonces, son una amenaza para el país y para su capacidad de emplear argentines. De esta manera, a partir de la separación entre nosotres y elles, y la repetición de los verbos "competir" y "necesitar" en referencia al ámbito del trabajo, Macri evoca el discurso dominante (Melella 2015) sobre les migrantes que plantea que les extranjeres que vienen a la Argentina les quitan el trabajo a les natives. Parecería que no es posible que haya trabajo para todas las personas del mundo: que algunos tengan trabajo implica, en el discurso del expresidente, que ese trabajo se le quite a otra persona. Esta misma representación se repite en otro discurso activando, además, el marco conceptual de la guerra:

(15) Porque en esa mesa vamos a defender y crear nuevo trabajo, trabajo que compite con nuestros hermanos de Latinoamérica, con los hermanos del mundo, porque todos quieren trabajo. En todas partes hay gente preocupada por conseguir un trabajo, no es sólo nuestra preocupación: en Asia, en Europa, en Estados Unidos, en América Latina (Discurso sobre el veto de la Ley de emergencia ocupacional, 20/05/2016) (cursivas nuestras).

Así, por más que haya una estrategia de acercamiento, "hermanos", el vínculo con les extranjeres sigue siendo de defensa.

\title{
5. Consideraciones finales
}

En este trabajo, analizamos las representaciones que el expresidente Mauricio Macri construye sobre les migrantes durante su primer año de mandato. Observamos que existe un silencio con respecto a las personas migrantes que viven en Argentina. Macri evita hablar de "migrantes" o "extranjeres" y reserva el término "inmigrantes" para referirse a acontecimientos del pasado: las personas que llegaron desde Europa a fines del siglo XIX y durante la primera parte del siglo XX. Para situaciones actuales, utiliza referencias abstractas como "comunidad" y eufemismos como "habitantes de otras partes del mundo". 
Una serie de metáforas se asocian de manera recurrente con les migrantes. La Argentina es conceptualizada como un país de inmigrantes y se activa el lugar común del crisol de razas a partir de la insistencia en la diversidad. También observamos la utilización de la frase hecha "hacer la América" para asociar la inmigración con un fenómeno transcontinental, algo que no se sostiene en el presente. Las metáforas de guerra construyen a les extranjeres como un enemigo que hay que combatir. Por último, y asociado con lo bélico, se encuentra la metáfora del país como un contenedor ${ }^{10}$ de una especie en particular: un edificio o, más específicamente, una casa. Toda casa tiene un adentro y un afuera, y puertas que pueden permanecer abiertas o cerradas. Así, cuando se trata de inversiones, las puertas se encuentran abiertas y les extranjeres son bienvenides; pero cuando se considera que constituyen un peligro o una amenaza, las puertas se cierran y se expulsa a quien las haya traspasado.

Les extranjeres son valorades a partir de lo que aportan al país. Así, las inversiones de empresas extranjeras o les trabajadores de países desarrollados que quieran contribuir con el crecimiento de la Argentina son bienvenides; mientras que el expresidente se desentiende de su responsabilidad por las personas que necesitaran del sistema de salud argentino o de un empleo, las cuales constituirían una competencia para les natives por recursos que se representan como escasos.

Además, Macri asocia a les extranjeres con el narcotráfico a partir del marco conceptual de la guerra: es necesario combatir la amenaza exterior. La seguridad en las fronteras terrestres (no marítimas o aéreas) implica que el peligro proviene de Latinoamérica. La disimilación con el resto del continente, e incluso con la población indígena de Argentina, aparece no solo de manera explícita, sino a través de la insistencia en la filiación europea de les argentines. Coincidimos con Oteiza, Novick y Aruj (2000) en su afirmación de que la idealización de una identidad europeizante es la contracara del discurso xenófobo.

En resumen, a pesar de los intentos por ser políticamente correcto al evitar términos que podrían tomarse como discriminatorios, observamos rasgos xenófobos que atraviesan el discurso de Macri, puesto que utiliza estrategias de disimilación para separar a les argentines de otros grupos de personas y se desentiende de los problemas de estos últimos. Les otres son una amenaza para les argentines ya sea porque se les asocia con el narcotráfico o la escasez de trabajo. Por último, las únicas referencias del ex presidente a inmigrantes - ya sea las migraciones europeas del pasado o aquellas confundidas con inversiones-demarcan a ciertas nacionalidades como deseables y, por lo tanto, aunque no lo diga, excluye a otras.

10 Conceptualizar a un país como un contenedor significa que hay un adentro, un afuera y un límite. También supone que el contenedor tiene una capacidad limitada, se puede llenar. Por ello, las metáforas bélicas suelen encontrarse asociadas a las metáforas de contenedores en el discurso racista: es necesario defender los límites del contenedor, evitar que entren intrusos o que se llene. 


\section{Bibliografía}

"Cardelli, Marina. 2018. “La reacción conservadora. Algunas preguntas teóricas en torno al debate sobre el plural del español y la aparición de la 'e' como práctica de lenguaje inclusivo en Argentina". Revista Entornos 31(1).

»Chilton, Paul. 2005. Missing links in mainstream CDA: Modules, blends and the critical instinct. A New Research Agenda in (Critical) Discourse Analysis: Teory, Methodology and Interdisciplinarity. editado por Ruth Wodak y Paul Chilton,. Amsterdam: John Benjamins.

" Chilton, Paul. 2011. "Still something missing in CDA". Discourse Studies13(6).

»Chilton, Paul. 2014. Language, Space and Mind: The Conceptual Geometry of Linguistic Meaning. Cambridge: Cambridge University Press.

»Discepolo, Armando. [1925] 2006. Babilonia. Una hora entre criados. Buenos Aires: Galerna.

》Domenech, Eduardo. 2009. "La visión estatal sobre las migraciones en la Argentina reciente: De la retórica de la exclusión a la retórica de la inclusión”. En Migración y política: el Estado interrogado. Procesos actuales en Argentina y Sudamérica, compilado por Eduardo Domenech, 21-70. Córdoba: UNC.

"Fairclough, Norman. 1992. Discourse and Social Change. Cambridge: Polity Press.

» Fairclough, Norman. 2003. Analysing Discourse. Textual Analysis for Social Research. London: Routledge.

" Fairclough, Norman. 2014. Language and Power. London: Routledge.

"Flax, Rocío. 2013. “Una aproximaciónal Análisis Crítico del Discurso”. En Alternativas epistemológicas para las ciencias sociales: ontología, lenguaje y política, editado por Silvia Rivera. Buenos Aires: Prometeo.

» Flax, Rocío. 2019a. "La criminalización de la inmigrante en el decreto 70/2017: un aporte desde el análisis del discurso". Revista de Lingüística Aplicada 57 (1).

" Flax, Rocío. 2019b. "La categorización del inmigrante en el discurso oficial argentino". Trabajo presentado en el congreso III DiscourseNet -ALED: Knowledge and Powerina Polycentric World, París, 11 a 14 de septiembre.

» Fowler, Robert, Robert Hodge, Gunther Kress y Tony Trew. 1983.Lenguaje y control. México: Fondo de cultura económica.

» Golay, Isabel. 2013.“Argentina 'crisol de razas':ficción y realidad”. Trabajo presentado en las VII Jornadas de Jóvenes Investigadores, Buenos Aires, 6-8 de noviembre.

» Halliday, M. A. K. y Christian Matthiessen. 2014. An introduction to functional grammar. $3^{\text {a }}$ edición. Routledge: Londres.

"Hart, Christopher. 2010.Critical Discourse Analysis and Cognitive Science New Perspectives on Immigration Discourse. London: Palgrave Macmillan.

" Hart, Christopher. 2014. Discourse, Grammar and Ideology. London: Bloomsbury.

" Hodge, Robert y Gunther Kress. 1993. Language as Ideology. London: Routledge \& Kegan Paul Books.

"Kaplan, Nora. 2004. “Nuevos desarrollos en el estudio de la evaluación en el lenguaje: La Teoría de la Valoración”. Boletín de Lingüística 22: 52-78. 
" Lakoff, George. 1987. Women, Fire and Dangerous Things. Chicago/London: University of Chicago Press.

» Lakoff, George. 2002. Moral Politics. How Liberals and Conservatives Think. Chicago/London: The University of Chicago Press.

» Lakoff, George. 2010. “Why it Matters How We Frame the Environment”. Environmental Communication 4 (1).

» Lakoff, George y Mark Johnson. 2003. Metaphors we live by. Chicago/London: The University of Chicago press.

» Martin, James y Peter White. 2005. The language of evaluation. Appraisal in English. New York: Palgrave Macmillan.

» Martínez Romagosa, Maite. y Flax, Rocío. 2020. Desalienar el ACD: Una revisión de la noción de ideología para devolver la crítica marxista al Análisis Crítico del Discurso. Pensamiento al margen, 12.

»Melella, Cecilia. 2015.“Migraciones latinoamericanas y prensa gráfica. Análisis comparativo entre Argentina y España”. Odisea. Revista de Estudios Migratorios 2.

» Moliner, María. 2007. Diccionario del uso del español. Madrid: Gredos.

»Oteiza, Enrique, Susana Novick y Roberto Aruj. 200o.Inmigracióny discriminación. Políticas y Discursos. Buenos Aires: Grupo Editor Universitario.

» Pérez, Sara Isabel. 2013. “Tecnologías digitales, análisis del discurso y multimodalidad: de la lingüística crítica a la semiótica social”. En Revista de ciencias sociales 5(23).

» Pérez, Sara Isabel (ed.). 2014. Análisis del discurso político. Mendoza:Editorial FFyL-UNCuyo.

» Reisigl, Martin y Ruth Wodak. 2001. Discourse and discrimination: Rhetorics of racism and antisemitism. Routledge: Londres.

»Sarlo, Beatriz y Santiago Kalinowski. 2019. La lengua en disputa. Un debate sobre el lenguaje inclusivo. Buenos Aires: Ediciones Godot.

»Soria, Sofía. 2009. "Las migraciones y el discurso multi/intercultural del estado en Argentina”. En Migración y política: el Estado interrogado. Procesos actuales en Argentina y Sudamérica, editado por Eduardo Domenech, 103-138. Córdoba: UNC.

»Stang, María Fernanda. 2009. “El migrante unidimensional. El dispositivo jurídico migratorio en la Comunidad Andina de Naciones”. En Migración y política: el Estado interrogado. Procesos actuales en Argentina y Sudamérica, editado por Eduardo Domenech, 301-354. Córdoba: UNC.

»Van Dijk, Teun. 1998. Ideology: A Multidisciplinary Approach. London: Sage.

»Wodak, Ruth. 2003. “El enfoque histórico del discurso”. En Métodos de análisis crítico del discurso, editado por Ruth Wodak y Michael Meyer. Barcelona: Editorial Gedisa. 